\section{$\underset{\substack{\text { hommes } \\ \text { \& migrations }}}{ }$}

\section{Hommes \& migrations}

Revue française de référence sur les dynamiques

migratoires

1307 | 2014

L'Afrique qualifiée dans la mondialisation

\title{
Le Quartier latin sénégalais à la croisée des chemins
}

\section{Mohamadou Sall}

\section{(2) OpenEdition}

\section{Journals}

\section{Édition électronique}

URL : http://journals.openedition.org/hommesmigrations/2879

DOI : 10.4000/hommesmigrations.2879

ISSN : 2262-3353

\section{Éditeur}

Musée national de l'histoire de l'immigration

\section{Édition imprimée}

Date de publication : 1 juillet 2014

Pagination : 57-65

ISBN : 978-2-919040-28-5

ISSN : $1142-852 X$

\section{Référence électronique}

Mohamadou Sall, «Le Quartier latin sénégalais à la croisée des chemins », Hommes \& migrations [En ligne], 1307 | 2014, mis en ligne le 01 juillet 2017, consulté le 03 mai 2019. URL : http://

journals.openedition.org/hommesmigrations/2879; DOI : 10.4000/hommesmigrations.2879 


\title{
LE QUARTIER LATIN SÉNÉGALAIS À LA CROISÉE DES CHEMINS
}

par MOHAMADOU SALL, maître de conférences en démographie, Institut de population, développement et santé de la reproduction (IFRPDSR), université de Dakar.

\author{
En septembre 2014, la énième grève des étudiants \\ de I'université Cheikh-Anta-Diop de Dakar pour \\ le non-paiement de leur bourse témoigne de la crise qui \\ secoue l'enseignement supérieur sénégalais. Pourtant, \\ le Sénégal demeure une destination stratégique et attractive \\ pour les étudiants francophones. La notoriété de cette \\ université, le développement d'écoles privées spécialisées \\ et la multiplication des accords d'équivalence des diplômes \\ avec les universités d'Europe ou d'Amérique du Nord \\ sont autant de moyens de promouvoir des formations répondant \\ aux besoins et aux codes du marché.
}

\begin{abstract}
Aujourd'hui, la crise qui secoue l'espace universitaire sénégalais depuis la rentrée académique d'octobre 2013 semble atteindre son paroxysme ${ }^{1}$. Cette crise serait pour certains indissociable de la massification des universités. L'université de Dakar, qui comptait en 19591300 étudiants², en accueillerait aujourd'hui près de 80 000. Mieux, les flux de nouveaux bacheliers connaîtront prochainement une nette augmentation. De façon générale, l'explosion de la demande d'accès à l'enseignement supérieur en Afrique noire est un fait marquant du secteur ${ }^{3}$. En Afrique subsaharienne, cette explosion engendre de nouveaux défis, du
\end{abstract}

fait de l'avancée dans la transition démographique et de la modification de la structure par âge de la population. Et pourtant, elle contraste avec l'insuffisance de l'offre dans le domaine, qui renvoie, d'une part, aux faibles capacités d'accueil des structures (amphithéâtres, salles de travaux dirigés et pratiques), au dénuement des campus (pavillons et autres lieux d'hébergement) et, d'autre part, à l'insuffisance du personnel enseignant. À cela s'ajoute le manque de diversification des filières de formation pour une demande de plus en plus exigeante. Ce décalage entre l'offre et la demande d'enseignement supérieur serait au cœur des crises cycliques 
que connaît le système universitaire sénégalais, obligé de se réajuster pour retrouver son lustre d'antan et faire face à la forte concurrence émanant des instituts d'enseignement supérieur privé. L'enjeu est de taille. Le repositionnement des universités publiques devrait leur permettre de bénéficier des retombées positives de l'augmentation des flux de migration estudiantine Sud-Sud. Il s'agit d'analyser ici l'évolution du pôle éducatif sénégalais à l'aune de ces mutations. Les éléments qui serviront à documenter en partie cette évolution sont tirés d'une analyse de données qualitatives recueillies auprès d'un échantillon de 70 étudiants inscrits à l'université Cheikh-Anta-Diop de Dakar (Ucad) et dans trois instituts privés de Dakar : le Centre africain d'études supérieures en gestion (Cesag), l'Institut africain de management (IAM) et l'Institut supérieur de management. Ces 70 étudiants ont été sélectionnés au hasard et la participation à l'enquête était libre. Ils ont été interviewés à l'aide d'entretiens semi-structurés.

\section{Le pôle éducatif sénégalais à l'aune d'un nouveau contexte}

Le pôle éducatif sénégalais est en partie un legs colonial. En effet, durant la période coloniale, le Sénégal revendiquait, tout comme le Dahomey (aujourd'hui le Bénin), cette appellation de Quartier latin africain qui, à l'instar de cet espace autour de la Sorbonne, symbolisait le lieu par excellence de production du savoir. L'histoire de l'enseignement au Sénégal est indissociable de celle de la création de l'Afrique-Occidentale française (AOF). L'ouverture de la première école à Saint-Louis en $1903^{4}$ suivie en 1912 de celle de l'École de médecine de Dakar et, en 1922, de l'École des enfants de troupe, allait former un embryon scolaire et universitaire, considéré comme la pépinière qui a fourni les premiers cadres aux colonies (instituteurs, infirmiers, médecins, vétérinaires, sous-officiers et officiers). Certains de ces cadres ont joué un rôle important dans le processus de décolonisation et présidé ensuite aux destinées de leur pays à l'indépendance.

Dans un article intitulé "Quelle capitale pour la littérature de langue française ?”, Mohamadou Kane montre que la ville de Saint-Louis a été jusqu'en 1930 la première capitale de la littérature africaine moderne, avant d'être supplantée par Paris et Dakar ${ }^{5}$. L'auteur soutient également le rôle majeur joué par la France dans l'implantation d'une infrastructure culturelle moderne à Dakar. Le rayonnement culturel du Quartier latin sénégalais va se renforcer avec la création en 1949 d'un certificat de physique, de chimie et de biologie au sein d'un Institut des hautes études de Dakar. Il est parachevé par la création en 1957 à Dakar de la 18e université française rattachée aux universités de Paris et de Bordeaux, et conçue selon les mots de son recteur d'antan "comme une université française au service de l'Afrique". L'accès à l'indépendance des pays de l'AOF au début des années 1960 bouleverse les choses. Les différents États indépendants mettent en place des structures d'enseignement pour doter leur administration d'agents et de cadres dont ils ont besoin. Cependant, malgré cette nouvelle donne, le Sénégal mantient son rôle majeur dans la formation des ressortissants des pays africains d'expression francophone. Baptisée en 1987 université Cheikh-Anta-Diop, l'institution continue d'accueillir des étudiants étrangers comme l'atteste le tableau ci-contre.

\section{L'Ucad face à l'augmentation de la mobilité internationale}

L'examen des statistiques fournies par la direction des systèmes d'information de l'Ucad montre l'importance relative des étudiants étrangers. Ces étudiants sont présents au niveau de toutes les facultés et de tous les instituts. Cependant, c'est la faculté de médecine, de pharmacie et d'odontologie (FMPO) qui accueille le plus d'étrangers, sui- 
$/ / /$ Tableau $n^{\circ} 1$ : Répartition des étudiants étrangers par faculté ou par institut entre 2008 et 2014.

\begin{tabular}{|c|c|c|c|c|c|c|}
\hline \multirow[b]{2}{*}{ ÉTABLISSEMENT } & \multicolumn{6}{|c|}{ ANNÉE UNIVERSITAIRE } \\
\hline & $\begin{array}{l}2008 \\
2009\end{array}$ & $\begin{array}{l}2009 \\
2010\end{array}$ & $\begin{array}{l}2010 \\
2011\end{array}$ & $\begin{array}{l}2011 \\
2012\end{array}$ & $\begin{array}{l}2012 \\
2013\end{array}$ & $\begin{array}{l}2013 \\
2014\end{array}$ \\
\hline $\begin{array}{l}\text { Centre d'études } \\
\text { des sciences et techniques } \\
\text { de l'information (CESTI) }\end{array}$ & 32 & 31 & 22 & 24 & 17 & 25 \\
\hline $\begin{array}{l}\text { École des bibliothécaires et } \\
\text { archivistes de Dakar (EBAD) }\end{array}$ & 22 & 107 & 108 & 44 & 7 & 47 \\
\hline $\begin{array}{l}\text { École normale supérieure } \\
\text { d'enseignement technique } \\
\text { et professionnel (ENSETP) }\end{array}$ & & 2 & 4 & 77 & 72 & 64 \\
\hline $\begin{array}{l}\text { École supérieure } \\
\text { d'économie appliquée } \\
\text { de Dakar (ESEA) }\end{array}$ & & & & 153 & 116 & 96 \\
\hline $\begin{array}{l}\text { École supérieure } \\
\text { polytechnique (ESP) }\end{array}$ & 98 & 80 & 90 & 74 & 59 & 84 \\
\hline $\begin{array}{l}\text { Faculté des sciences } \\
\text { économiques et de gestion } \\
\text { (FASEG) }\end{array}$ & 486 & 527 & 530 & 368 & 362 & 361 \\
\hline $\begin{array}{l}\text { Faculté des sciences et } \\
\text { technologies de l'éducation } \\
\text { et de la formation (FASTEF) }\end{array}$ & 42 & 40 & 29 & 10 & 9 & 3 \\
\hline $\begin{array}{l}\text { Faculté des lettres et } \\
\text { sciences humaines (FLSH) }\end{array}$ & 215 & 201 & 235 & 173 & 157 & 204 \\
\hline $\begin{array}{l}\text { Faculté de médecine } \\
\text { de pharmacie } \\
\text { et d'odontologie (FMPO) }\end{array}$ & 2486 & 1785 & 1877 & 2514 & 2777 & 2939 \\
\hline $\begin{array}{l}\text { Faculté des sciences } \\
\text { juridiques et politiques } \\
\text { (FSJP) }\end{array}$ & 371 & 332 & 428 & 442 & 421 & 306 \\
\hline $\begin{array}{l}\text { Faculté des sciences } \\
\text { et techniques (FST) }\end{array}$ & 415 & 455 & 434 & 438 & 455 & 491 \\
\hline $\begin{array}{l}\text { Institut de formation et } \\
\text { de recherche en population, } \\
\text { développement et santé de } \\
\text { la reproduction (IFRPDSR) }\end{array}$ & 14 & 14 & 13 & 17 & 11 & \\
\hline $\begin{array}{l}\text { Institut national supérieur } \\
\text { de l'éducation populaire } \\
\text { et du sport (INSEPS) }\end{array}$ & 33 & 41 & 26 & 36 & 40 & 24 \\
\hline Total UCAD & 4214 & 3615 & 3796 & 4370 & 4503 & 4644 \\
\hline
\end{tabular}


vie de la faculté des sciences et techniques (FST), de celle des sciences juridiques et politiques et, enfin, de la faculté des sciences économiques et de gestion. Sur les 4644 étudiants inscrits en 20132014, 2939 l'étaient à la faculté de médecine, soit $63 \%$ des étrangers inscrits.

La faculté de médecine, de pharmacie et d'odontologie s'est toujours distinguée par le nombre d'étudiants en provenance du Maghreb en général et du Maroc en particulier. Aujourd'hui, beaucoup d'étudiants issus de cette région y sont encore inscrits. On note aussi la présence d'étudiants en provenance des pays francophones de l'Afrique subsaharienne qui viennent poursuivre des études de spécialisation médicale. Ce pôle éducatif sénégalais essentiellement porté par l'université de Dakar a cherché à demeurer une référence en Afrique subsaharienne dans les deux décennies qui ont suivi les indépendances. L'université Cheikh-Anta-Diop de Dakar, qui a connu sa première année blanche en 1988, renoue avec la crise au cours de l'année académique 1993-1994, déclaLa faculté de médecine, rée “année invalide”. Cette de pharmacie et période est tout à fait sind'odontostomatologie s'est

toujours distinguée par le nombre d'étudiants en provenance du Maghreb en général et du Maroc en particulier gulière car elle s'inscrit dans un contexte particulier marqué par des faits majeurs qui vont amener le pôle éducatif sénégalais à se repositionner sur le champ de l'enseignement supérieur.

Le premier a trait à l'augmentation des flux de migration estudiantine au cours de ces dernières décennies ${ }^{6}$, une des formes majeures de la mobilité internationale $e^{7}$ Selon les estimations de l'Unesco, à la fin des années 2000, le volume de la mobilité estudiantine se chiffrait à plus de 2,8 millions de personnes, soit une augmentation de $53 \%$ par rapport à 2000. En outre, à l'horizon 2025, on estime que 7,2 millions de personnes poursuivront leurs études à l'étranger, soit une progression de 188 $\%$ par rapport à $2006^{\circ}$. Cette progression touche aussi les pays en développement. Deux faits l'expliquent. Entre 1962 et 1998, les étudiants provenant des pays en développement ont représenté plus de $60 \%$ du pourcentage total des étudiants inscrits hors de leur pays d'origine ${ }^{9}$. Aussi, analyser le repositionnement du pôle éducatif sénégalais en rapport avec l'augmentation des flux de migration estudiantine, c'est aussi, d'une certaine manière, "penser l'Afrique à l'aune des globalités émergentes ${ }^{10 "}$. Le second fait est lié aux conséquences de la dévaluation du franc CFA en 1994 qui a amenuisé les possibilités financières de certains parents d'élèves et d'étudiants qui souhaitaient envoyer leurs enfants poursuivre leurs études en Europe ou aux États-Unis d'Amérique, et les a obligés à envisager des solutions alternatives. Dans ce contexte, les pays qui développent un système d'enseignement moderne peuvent devenir très compétitifs.

\section{L'irruption des structures d'enseignement privé}

Au début des années 1990, Dakar recommence à être le pôle de convergence des étudiants en provenance des pays francophones d'Afrique de l'Ouest désireux de poursuivre des études supérieures. Cette période coïncide avec l'arrivée sur le marché de l'éducation des instituts d'enseignement supérieur privé. Aux sources de cette irruption, on note un parrainage de l'État. En son article 3, la loi 91-22 du 16 février 1991 dispose que "l'initiation privée, individuelle ou collective, peut, dans les conditions définies par la loi, concourir à l'œuvre d'éducation et de formation". En réalité, l'attitude de l'État envers l'enseignement supérieur privé est assez

6. Allan M Findlay, Russell King, Fiona M Smith, Alistair Geddes, Ronald Skeldon, "World class? An investigation of globalization, difference and international student mobility”, in Transactions, vol. 37, n 1, 2012, pp. 118-131. 7. Voir Rajika Bhandari, Peggy Blumenthal, "Global student mobility in international institute for education", New York, éd. Higher education on the move, IIE, 2009, pp. 1-15, cités par Allan M. Findlay et al., op. cit. 8. Philip G. Altbach, Liz Reisberg, Laura E. Rumbley, “Trends in global higher education. Tracking an academic revolution”, a report prepared for the Unesco 2009 world conference on higher education, p. 25. 9. Abdelkader Latreche, "Les migrations étudiantes de par le monde”, in Hommes \& Migrations, $n^{\circ}$ 1233, 2001, p. 14). 10. Sandrine Perrot, Dominique Malaquais, “Penser l'Afrique à l'aune des globalisations émergentes", in Politique africaine, $n^{\circ} 113,2009$, pp. 5-27. 
équivoque. De plus, elle oscille entre une ferme volonté de contrôler l'action de ces structures et une invite à leur faire jouer un rôle dans la prise en charge d'une demande d'enseignement supérieur de plus en plus forte. Ainsi, au cours des rentrées académiques 2012-2013 et 2013-2014, le ministère de l'Enseignement supérieur a posé un acte de rupture en demandant aux écoles et aux universités privées d'accueillir les bacheliers qui ne pouvaient pas être orientés dans les différentes universités publiques, engorgées.

Excepté le cas du Centre africain d'études supérieures en gestion (Cesag) créé en 1985, la plupart des instituts privés du supérieur ont vu le jour au cours de la décennie 1990. L'Institut supérieur de management (ISM), le premier institut d'enseignement privé, a été créé en 1992. Un an plus tard, on note l'ouverture de Sup de Co, une haute école commerciale. Au cours de la même année, est créée une école privée dénommée AFI-Université de l'entreprise. En 1996, l'Institut africain de management a ouvert ses portes. C'est en 1995 que la première université privée, l'université Dakar-Bourguiba, a été ouverte par un ancien professeur de mathématiques de l'Ucad. L'université du Sahel lui emboîte le pas en 1998. En 2003, la première faculté de médecine privée accueille ses étudiants. Au cours de l'année académique 2006-2007, la benjamine des universités privées, l'université Amadou-Hampaté-Ba, est ouverte.

\section{Inventer de nouvelles formes de mobilité pour les étudiants}

En Afrique del'Ouest, Dakarsepositionne commela destination principale des étudiants provenant des paysfrancophones. Cetteattraction peuts'expliquer

par l'image de modernité véhiculée surtout par les instituts d'enseignement supérieur privés qui proposent des offres alléchantes et compétitives.

Le rêve des jeunes élèves et des étudiants des pays africains a été, et demeure toujours, d'aller poursuivre leurs études dans les pays développés (Europe et Amérique du Nord) afin d'y obtenir "un diplôme reconnu sur le plan local et international pour trouver plus facilement un emploi ${ }^{11 "}$. Cependant, il devient difficile de concrétiser ce projet dans la mesure où les conditions qui organisent la venue des étudiants dans ces pays semblent être de plus en plus contraignantes. Serge Slama

C'est en 1995 que

la première université privée, a caricaturé ces conditions en évoquant une "impossible admission ${ }^{12 "}$ des étrangers et a montré comment la procédure d'"admission préalable" mise en place en 1981 et initialement destinée à mieux gérer les flux d'étudiants entrant a été dévoyée. Selon Slama, cette procédure serait "utilisée aujourd'hui par les pouvoirs publics comme un 'rouage' dans un processus d'endiguement des entrées d'étrangers en premier et en deuxième cycles universitaires et de régulation des flux". En fait, la progression du nombre d'étudiants africains en France doit plutôt être analysée en termes d'évolution de la structure démographique de ces pays émetteurs de migrants étudiants et non en termes de diminution des contraintes en matière d'admission.

Les instituts ont donc cherché à réaliser le rêve de ces élèves et étudiants désireux de poursuivre leurs études en Europe et en Amérique du Nord en leur offrant, à travers la codiplômation ${ }^{13}$, la possibilité de rester au Sénégal tout en obtenant un diplôme portant le sceau d'une université ou d'une école supérieure occidentale. Une autre composante attractive du portefeuille de services offerts est

11. Magatte Fall, “Migration des étudiants sénégalais. Impact sur le développement de leur pays d'origine”, in Hommes \& Migrations, $\mathrm{n}^{\circ}$ 1286-1287, juillet-octobre 2010, pp. 226. 12. Serge Slama, La Fin de l'étudiant étranger, Paris, L'Harmattan, 1999, pp. $179-181$. 13. L'IAM délivre des diplômes en collaboration avec des universités suivantes : l'université du Québec à Chicoutimi au Canada, l'université de Strasbourg et de Mulhouse en France. Quant à l'Institut supérieur de management (ISM), il fait de la codiplômation avec des écoles privées de management françaises : École supérieure pour le développement économique et social (ESDES) Lyon, Rouen Business School, Reims Management School. Cependant, c'est la Bordeaux Management School qui, malgré une arrivée tardive sur le marché (2008), est allée plus loin dans la codiplômation. En fait, la maison mère de Bordeaux Management School a tout simplement délocalisé une partie de son programme de formation à Dakar. Le cursus des étudiants est scindé en deux parties, la première au Sénégal et la seconde à Bordeaux. 
le programme de préparation aux grandes écoles françaises de commerce. Les écoles privées ont très tôt senti tout le bénéfice qu'elles pouvaient tirer du développement d'un programme de formation particulièrement prisé comme la prépa HEC. En 2004, HEC Paris Une autre composante a établi un accord de parteattractive du portefeuille de services offerts est le programme de préparation aux grandes écoles françaises de commerce. nente à Dakar. Une décennie plus tard, le Groupe Sup de Co de Montpellier Business School lui emboîte le pas, accréditant la pertinence de ce choix stratégique qui consiste à préparer des étudiants à poursuivre leurs études en France ${ }^{14}$. Dans ce même registre, le hub éducatif dakarois doit son attractivité au fait d'être, avec la ville de Casablanca, l'un des deux centres africains qui abritent annuellement des épreuves de préparation et/ou d'examen du concours français Passerelle, un grand concours d'épreuves communes en France. Ce concours est organisé conjointement par seize écoles supérieures de commerce de l'Hexagone.

\section{Adapter les cursus aux besoins du marché}

Les écoles privées proposent des cursus essentiellement orientés vers le management. L'examen de l'échantillon montre l'intérêt pour les études dans ce domaine. Sur les 47 étudiants interviewés au niveau des trois instituts (Cesag, IAM et Institut supérieur de management), 35 poursuivent de telles études. La moyenne d'âge des étudiants enquêtés est de 24 ans. Cet âge avancé rend compte du profil de l'évolution de la demande d'en- seignement supérieur en Afrique. Des indépendances jusqu'à la fin des années 1980, l'université Cheikh-Anta-Diop de Dakar avait le monopole de l'enseignement supérieur. La moyenne d'âge à l'entrée tournait autour de 20 ans et les études les plus longues étaient généralement les études médicales. Dans les autres facultés (sciences, lettres et sciences humaines, droit et sciences économiques), l'enrôlement des étudiants dans les programmes doctoraux était assez marginal. Il était exceptionnel d'y retrouver des étudiants âgés de plus de 40 ans. Mieux, de façon générale, l'université de Dakar n'avait pas pour vocation de fournir un enseignement professionnalisant à des travailleurs ayant acquis sur le terrain une expérience pratique. Livrant son expérience d'ancien recteur de l'Ucad, Abdou Salam Sall souligne : "L'offre de formation dans les facultés n'avait aucune articulation avec les besoins du marché, mis à part la faculté de médecine, de pharmacie et d'odontostomatologie et, dans une moindre mesure, la faculté de droit ${ }^{15}$." Le Centre africain d'études supérieures en gestion (Cesag) créé en 1985 par la Conférence des chefs d'État de la communauté économique de l'Afrique de l'Ouest (CEAO) décide d'offrir cette formation à des professionnels désireux de parfaire leur formation en vue de saisir de nouvelles opportunités sur le marché de l'emploi. Les 16 nationalités représentées dans l'échantillon témoignent à souhait que Dakar est devenu un hub éducatif régional. Les étudiants proviennent essentiellement des pays francophones de l'Afrique occidentale et centrale (Côte d'Ivoire, Bénin, Burkina Faso, Niger, Gabon, Centrafrique, Tchad, Cameroun, Congo, Congo RDC). On retrouve aussi des étudiants provenant d'autres pays francophones (Comores et Mauritanie). Le caractère panafricain du hub dakarois transparaît à travers la présence d'étudiants provenant de pays lusophones (Cap-Vert et Guinée-Bissau). 


\section{La concurrence entre institutions}

Les motivations invoquées par les étudiants interviewés à l'université Cheikh-Anta-Diop et dans les instituts privés sont diverses. Certaines sont marginales : elles vont de la recherche d'une filière absente dans les offres de formation du pays d'origine à la stabilité politique du Sénégal, en passant par l'usage du français comme langue d'enseignement, ou la découverte de la culture et de l'art de vie sénégalais. En revanche, les motivations principales sont d'abord la notoriété et, ensuite, la présence familiale au Sénégal. 39 étudiants sur 70, soit plus d'un étudiant sur deux, convoquent la notoriété de l'établissement.

Les informations concernant les instituts sont obtenues à travers trois principaux canaux : Internet, les amis et les parents. La notoriété des instituts privés doit beaucoup au marketing, qui, au sein de l'enseignement supérieur, a revêtu une importance capitale au moment où la globalisation touchait le secteur de l'éducation ${ }^{16}$. Cependant, toutes les universités ne sont pas en mesure d'opérer un marketing dont l'absence contribue, d'ailleurs, à les desservir ${ }^{17}$. Cela a été le cas, pendant longtemps, de l'université Cheikh-Anta-Diop qui n'a pas véritablement développé une stratégie de marketing qui lui aurait permis d'attirer une nouvelle catégorie d'étudiants davantage intéressés par des formations professionnelles. Or la vision moderne de l'université préconise la mise en place de "systèmes ouverts qui reconnaissent l'expérience pertinente acquise antérieurement, les équivalences de diplômes, le transfert des unités de valeur, les régimes de programmes d'enseignement, l'accès aux bourses nationales et aux prêts pour étudiants, et un cadre global de qualifications et d'apprentissage permanent ${ }^{18}$ ". C'est justement ce créneau appelé "Executive Program" que les instituts privés ont investi. Il s'agit de proposer à des catégories d'étudiants possédant déjà une expérience professionnelle des cursus de formation conformes à leurs attentes et leur permettant de mieux se positionner sur le marché de l'emploi.

À l'instar du pôle éducatif français où "le premier constat est celui d'une forte diversification des cursus offerts ${ }^{19}$ ", la donne éducative sénégalaise se caractérise par une forte demande d'enseignement supérieur. Celle-ci est diversifiée, au regard des pays d'origine et des caractéristiques sociales et professionnelles, et exigeante dans la sélection des cursus et des filières d'enseignement. Cette donne transforme l'enseignement supérieur en marché, où les offres deviennent concurrentielles. Toutes les études sont ouvertes à la concurrence : alors que les instituts privés comme Sup de Co, l'ISM, l'IAM, le Cesag et l'université Dakar-Bourguiba entrent en concurrence avec les facultés des sciences économiques et juridiques pour les formations dans le domaine du management, des finances et des professions judiciaires, les universités du Sahel et AmadouHampathé-Ba étendent la concurrence aux sciences humaines et sociales. En
Il s'agit de proposer à des catégories d'étudiants possédant déjà une expérience professionnelle des cursus de formation conformes à leurs attentes et leur permettant de mieux se positionner sur le marché de l'emploi. 2003, la faculté de médecine Saint-Christopher-Iba-Mar-Diop est venue faire sauter le verrou d'un domaine jusque-là réservé à l'enseignement supérieur public. Jusqu'à cette date, seule la faculté de médecine, de pharmacie et d'odontologie (FMPOS) de l'université Cheikh-Anta-Diop délivrait des diplômes de médecine. La rupture opérée par la faculté privée est radicale. En plus de mettre fin à l'exclusivité dans le domaine de la formation et de la délivrance du diplôme de médecine, elle mettait sur le marché 
une offre pédagogique différente et censée être plus attractive : une formation de médecin sur cinq années, soit deux ans de moins que la durée des études médicales à l'Ucad. Cette première faculté privée ouvrira la voie à un second institut privé : l'Institut privé de formation et de recherches médicales de Dakar (Ipformed-Imsd) démarre officiellement ses activités d'enseignement en 2012. Une centaine d'étudiants constitués à 80 \% étrangers y suivent les cours. Ces instituts privés forment avec l'université Cheikh-Anta-Diop la pierre angulaire du renouveau du Quartier latin sénégalais. À l'échelle de l'Afrique de l'Ouest et du centre, ils constituent la vitrine attractive d'un enseignement supérieur qui draine vers Dakar de plus en plus d'étudiants.

\section{Entretenir l'attractivité du pôle éducatif sénégalais}

Cette donne concurrentielle de l'enseignement supérieur privé est bien perçue au niveau de l'enseignement supérieur public. Le plan stratégique 2011-2016 de l'Ucad évoque explicitement "les problèmes de concurrence". Cette prise de conscience oblige l'enseignement supérieur public à rompre avec la conception humboldtienne de l'université, suivant en cela la rupture déjà opérée par les universités occidentales vers le milieu des années 1980 et au cours des années $1990^{20}$. Cette rupture serait conforme à la vision du New Policy Management qui repose sur des principes d'utilité sociale, d'impulsion de l'innovation technologique et d'accountability $^{21}$. Dans le registre des actions entreprises par l'Ucad, on note en effet la planification stratégique, l'augmentation des droits d'inscription et du prix des tickets-repas et la rationalisation des effectifs.

D'autres actions figurent aussi en bonne place comme la création d'une clinique médicale de pointe et la mise en place d'une réglementation de la fonction de services qui autorise la création d'ins- tituts privés de formation au sein de l'espace universitaire : l'Institut de formation en administration et création d'entreprises (Iface), le Centre de recherches et de formation en développement économique et social (Crefdes), l'École supérieure polytechnique (ESP). À ces actions, s'ajoutent la mise en place d'une fondation pour soutenir les investissements et identifier des "stratégies de financement alternatives sans l'intervention de l'État", le développement d'incubateurs d'entreprises au niveau de l'Ucad et de l'université Gaston-Berger de Saint-Louis (UGB), la signature avec la Banque mondiale de contrats de performance (CDP), ainsi que l'établissement de règles de transparence budgétaires.

Le ministère de l'Enseignement supérieur a proposé, dans un souci de transparence, que soit institué dans chaque université un guichet unique qui gérerait tous les fonds des programmes et des projets de recherche. Les universités s'inscrivent aussi dans une politique de décentralisation articulée autour de la création de pôles régionaux de formation et de recherche. Cette politique consiste, pour chaque université, à identifier des écoles supérieures qu'elle intègre : l'École nationale supérieure d'agriculture (ENSA), l'École polytechnique de Thiès (EPT) pour l'université de Thiès, l'École nationale des cadres ruraux (ENCR) pour l'université de Bambey et l'École nationale d'économie appliquée (ENEA) pour l'Ucad. Ces aménagements s'accompagnent de réformes didactiques comme la mise en œuvre de la réforme licence-master-doctorat (LMD), que le plan stratégique de l'Ucad 2011 2016 présente comme "une des réponses adaptées aux multiples défis de notre enseignement supérieur". De surcroît, le plan de l'Ucad mentionne que cette réforme "exprime ainsi la volonté de mieux se positionner dans un monde dont les caractéristiques modales sont la globalisation, la numérisation, l'économie de la connaissance, l'évolution rapide des sciences et des techniques, l'intégration régionale, et dans une Afrique engagée dans la lutte contre le sous-développement, les grandes endémies et pour l'intégration conti- 
nentale ${ }^{22 "}$. Les réformes concernent aussi l'alignement des cursus, de la pédagogie et du système d'accréditation sur les normes internationales, la création d'écoles doctorales articulées autour de laboratoires, l'utilisation massive des nouvelles technologies de l'information et de la communication (inscription en ligne des nouveaux bacheliers), la promotion d'un e-learning. Au même moment, l'université joue sur la préservation des domaines de souveraineté comme le privilège d'organiser l'internat de médecine malgré les tentatives visant à casser ce monopole.

Pourtant, pour certains chercheurs, les exercices d'ajustement auxquels se livre l'université sont imposés et conformes à une vision néolibérale de l'éducation. Cette vision commence à s'imposer au cours de la décennie 1980 et serait celle de "lajustement éducatif, impulsé par les réflexions de la Banque mondiale ${ }^{23 "}$. Dans la préface de l'ouvrage L'Afrique noire face à sa laborieuse appropriation de l'université. Les cas du Sénégal et du Cameroun, Jean-Marc Ela souligne la volonté de "soumettre les universités aux lois du marchée ", volonté conforme à la perception de la Banque mondiale qui considère que l'enseignement supérieur n'est pas un bien public mais privé et que les universités africaines sont maintenues dans un état traditionnel où elles ne seraient pas sensibles aux besoins des marchés. De son côté, l'enseignement supérieur privé use aussi de stratégies pour gagner des parts de ce marché en pleine expansion : la recherche effrénée et systématique de l'homologation des diplômes délivrés par le Conseil africain et malgache pour l'enseignement supérieur (Cames), l'utilisation à temps partiel des enseignants des universités publiques sénégalaises et étrangères pour assurer les charges d'enseignement et d'encadrement, la tentative de faire participer ses étudiants au concours élitiste de l'internat de médecine, l'investissement dans la recherche (écoles doctorales) et le développement d'accords de partenariats avec des écoles et universités étrangères.

\section{Conclusion}

L’avancée du Sénégal dans la transition démographique couplée aux stratégies visant la scolarisation universelle avant 2025 crée une demande d'enseignement revigorée par les flux en provenance des pays de l'espace francophone. Cette augmentation de la demande d'enseignement supérieur s'inscrit dans une temporalité qui est celle de la diversification des cursus de formation, des innovations dans la transmission des connaissances, de l'établissement de passerelles entre les lieux de production des savoirs et les lieux de leurs usages. Elle s'inscrit aussi dans des spatialités parfois virtuelles rendant possibles l'ubiquité dans l'acquisition et la transmission des savoirs et des savoir-faire. Ces éléments sont constitutifs d'une nouvelle donne qui transforme l'enseignement supérieur sénégalais en marché concurrentiel, où les offres éducatives des universités publiques sont obligées d'entrer en compétition avec celles de l'enseignement supérieur privé. Cette progression de la demande est aussi indissociable de l'augmentation des flux de migrations estudiantines, en particulier Sud-Sud.

La coexistence d'une université publique de renom, classée en 2013 première université en Afrique occidentale et centrale par International Colleges \& Universities, et d'instituts privés attire à Dakar des milliers d'étudiants et en fait un hub éducatif cosmopolite. Pour l'université, il faut impérativement prendre des parts de ce marché en pleine expansion. La question qui reste en suspens est de savoir si l'université publique sénégalaise, qui a connu ses heures de gloire en s'ajustant aux nouvelles lois du marché, pourra trouver un juste équilibre entre une alma mater pourvoyeuse de services à la communauté (nouveau credo de l'université Cheikh-Anta-Diop) et une efficacité optimale au sens de Pareto. 